\title{
Hypothalamus-Pituitary-Adrenal Axis Hypersuppression Is Associated with Gastrointestinal Symptoms in Major Depression
}

\author{
Pontus Karling, ${ }^{1 *}$ Mikael Wikgren, ${ }^{1,2}$ Rolf Adolfsson, ${ }^{2}$ and Karl-Fredrik Norrback ${ }^{2}$ \\ ${ }^{I}$ Department of Public Health and Clinical Medicine, Division of Medicine, Umeå University, Umeå, Sweden; and ${ }^{2}$ Department of Clinical \\ Sciences, Division of Psychiatry, Umeå University, Umeå, Sweden
}

\section{Background/Aims}

Gastrointestinal symptoms and hypothalamus-pituitary-adrenal (HPA) axis dysfunction are frequently observed in patients with major depression. The primary aim of the study was to investigate the relationship between HPA-axis function and self-perceived functional gastrointestinal symptoms in major depression.

\section{Methods}

Patients with major depression $(n=73)$ and controls representative of the general population $(n=146)$ underwent a weightadjusted very low dose dexamethasone suppression test (DST). Patients and controls completed the gastrointestinal symptom rating scale-iritable bowel syndrome (GSRS-IBS) and the hospital anxiety depression scale. Medical records of the patients were screened over a ten year period for functional gastrointestinal disorder and pain conditions.

\section{Results}

Patients with high GSRS-IBS scores (above median) exhibited HPA-axis hypersuppression more often than controls (defined by the lowest 10\% cutoff of the post-DST cortisol values among controls, adjusted OR 7.25, Cl 1.97-26.7) whereas patients with low GSRS-IBS scores did not differ from controls concerning their post-DST cortisol values. Patients who had consulted primary care for functional gastrointestinal disorder $(P=0.039)$, lumbago $(P=0.006)$ and chronic multifocal pain $(P=0.057)$ also exhibited an increased frequency of hypersuppression.

\section{Conclusions}

HPA-axis hypersuppression is associated with functional gastrointestinal symptoms in patients with major depression.

(J Neurogastroenterol Motil 2016;22:292-303)

\section{Key Words}

Depression; Dexamethasone; Hypocortisolism; Hypothalamo-hypophyseal system; Irritable bowel syndrome

Received: April 17, 2015 Revised: August 21, 2015 Accepted: September 13, 2015

(a) This is an Open Access article distributed under the terms of the Creative Commons Attribution Non-Commercial License (http://creativecommons. org/licenses/by-nc/4.0) which permits unrestricted non-commercial use, distribution, and reproduction in any medium, provided the original work is properly cited.

*Correspondence: Pontus Karling, MD, PhD

Department of Public Health and Clinical Medicine, Division of Medicine, Umeå University, SE-90187 Umeå, Sweden Tel: +46-907850000, Fax: +46-90143986, E-mail: pontus.karling@vll.se 


\section{Introduction}

Gastrointestinal (GI) symptoms, functional GI disorders, and a broad range of other somatic symptoms are common in patients with major depression, ${ }^{1-3}$ and comorbidity with somatic symptoms is associated with a more severe and resistant form of depression. ${ }^{4}$ Irritable bowel syndrome (IBS) patients also exhibit a high degree of comorbidity with depression and anxiety, possibly indicating shared mechanistic pathways connecting these disorders. ${ }^{5,6}$

The underlying physiology linking GI symptoms and affective disorders is however poorly understood; dysfunction of noradrenergic pathways, serotonergic pathways, the autonomic nervous system, and the hypothalamic-pituitary-adrenal (HPA) axis has been proposed as explanations. ${ }^{5,7,8}$ The present study focuses on the HPA-axis, which plays a central role in the neuroendocrine response to stress with cortisol being it's main effector constituent, since both IBS and depression have been reported to exhibit HPAaxis dysregulation and that stress-dysregulation has been suggested as one important etiologic factor in both conditions. ${ }^{9-18}$

While stress has traditionally been associated with increased cortisol levels and HPA-axis hyperactivity, there is a growing body of literature describing lowered cortisol levels and hypoactivity of the HPA-axis in a number of stress related disorders ${ }^{19-35}$; eg, posttraumatic stress disorder (PTSD), ${ }^{30,31}$ chronic fatigue syndrome, ${ }^{22-24}$ burnout, ${ }^{25,26}$ as well as in fibromyalgia and chronic somatic pain conditions of unknown or uncertain etiology. ${ }^{28,29,32-34}$ Dysfunction of the HPA-axis, including hypoactivity, has also been reported in IBS. ${ }^{9-11}$ The HPA-axis hypoactivity observed in stress-related conditions (including depression), is thought to develop out of chronic stress, where an initial stage of HPA-axis hyperactivity eventually evolves into a hypoactive one. ${ }^{19,35}$ This stress-induced HPA-axis hypoactivity is commonly denoted hypocortisolism (low cortisol levels and adrenal insufficiency) and the polar opposite state will be denoted hypercortisolism. The mechanistic underpinnings of hypocortisolism is however largely unknown. Early studies in depression, mainly investigating younger inpatients, typically reported the existence of an HPA-axis hyperactivity, ${ }^{12-14}$ whereas more recent studies in older outpatients have in addition to findings of hypercortisolism also discovered patients exhibiting hypocortisolism. ${ }^{15-18}$

An exaggerated negative feedback response (also denoted hypersuppression) of the HPA-axis as captured by a low cortisol value, post a low dose dexamethasone suppression test (DST), is believed to be the earliest and most common characteristic of hypocortisolism according to Fries et al. ${ }^{19}$ Measurements of the negative feedback sensitivity of the HPA-axis commonly employ different DSTs. The use of a low dose or a very low dose regimen (0.5 mg dexamethasone or lower) allows for the discrimination of hypersuppression from normal and decreased suppression (denoted here intermediate or normal suppression and hyposuppression, respectively). In contrast, conventional high dose DSTs (1.0 mg dexamethasone or higher) causes a cortisol "bottom effect," masking subjects with hypersuppression among subjects with normal suppression. ${ }^{31}$

Based on the above we felt it was warranted, for the first time, to explore the relationships between self-perceived GI symptoms (including diagnosis of functional GI disorders) and consulting behavior for functional GI disorder to the HPA-axis function, as captured by a very low dose DST, among patients with recurrent major depressive disorder. As a secondary aim, we decided to investigate the relationship between the consulting behavior for common pain conditions and the function of the HPA-axis in depression due to the co-assocation of a broad range of somatic conditions of uncertain etiology with both depression and HPA-axis dysregulation.

\section{Materials and Methods}

\section{Participants}

Eighty-two unrelated individuals diagnosed with major depressive disorder of recurrent type without other major psychiatric comorbidity were included in a study with multiple research outcomes. All patients were interviewed by a psychiatrist and fulfilled the Diagnostic and Stastical Manual of Mental Disorders (DSM)IV criteria for major depressive disorder, recurrent type with at least 2 well documented and medically treated major depressive episodes. ${ }^{36}$ All patients were outpatients living in the Umeå municipality, northern Sweden, and no changes in treatment had occurred for the last 3 months prior to the study. Psychiatric comorbidity that was excluded for: PTSD, anxiety disorders, substance and alcohol abuse, and life time occurrence of bipolar spectrum symptoms based on interview and medical records from the psychiatric clinic. Further exclusion criteria that could be mentioned were mental retardation, dementia, organic brain damage, serious visual or auditory handicaps, not having Swedish as a mother tongue, or any other feature that would compromise the ability to comply with the study protocol. We also excluded patients with an established GI disease or abdominal surgery within 3 months before or after the study. The aim of the study was to investigate the relationship between self-perceived GI symptoms and consulting behaviour for 
functional GI disorder to the HPA-axis function of patients with recurrent major depressive disorder, and therefore patients who had a diagnosis of functional GI disorders in the primary care were not excluded. Further, all patients on steroid therapy or antibiotics were excluded. This lead to a reduction of the patient sample from 82 to 73 subjects; 5 with a history of GI disease or recent bowel surgery, 3 taking steroid medications, and 1 taking antibiotics were excluded.

The control sample consisted of unrelated age and sex matched subjects ( $n=146$, two controls for each patient) not suffering from a diagnosed psychiatric condition and similarly were free from steroid and antibiotics medication, without an established GI disease or abdominal surgery within 3 months before or after the study. They were randomly selected and were part of the Betula study, a large prospective cohort study investigating various aspects of memory, health, and aging. ${ }^{37}$ The Betula participants are recruited by random selection from the population registry of Umeå, northern Sweden. Exclusion criteria for the controls were in addition similarly dementia, mental retardation, organic brain damage, serious visual or auditory handicaps, not having Swedish as a mother tongue, and any other feature that would compromise the ability to comply with the study protocol. The Betula study population has been validated to conform well with the general population of northern Sweden. More details on the Betula study can be found in Nilsson et al, 1997. ${ }^{37}$ Despite the matching the patients were slightly older than the controls, although not significantly (Table 1).

All participants within the present study were Caucasian and in addition to a clinical interview also underwent a physical examination including a limited clinical biochemical screen. All participants gave their consent and the study was approved by the The Regional Ethical Review Board in Umeå, Sweden.

\section{Dexamethasone Suppression Test}

All 73 patients and 146 controls underwent a weight-adjusted very low dose DST and were instructed to ingest a pre-measured dexamethasone solution at $11 \mathrm{PM}$ the night before blood draw. The DST has previously been employed in research on obesity but has also more recently been used by us in studies on affective disorder and general population samples. ${ }^{38-40}$ The solution contained $3.5 \mu \mathrm{g}$ dexamethasone per kilogram of body weight $(175 \mu \mathrm{g}$ and $280 \mu \mathrm{g}$ of dexamethasone for a person weighing $50 \mathrm{~kg}$ and $80 \mathrm{~kg}$, respectively). The following morning between $8 \mathrm{AM}$ and $10 \mathrm{AM}$ blood was drawn. Serum cortisol was analyzed in an accredited laboratory by Roche Elecsys reagents on a Modular E170 analyzer (coefficient of variance $[\mathrm{CV}]<10 \%)$. Subjects who had their plasma dexamethasone levels measured (all patients and 60 randomly selected controls) all exhibited detectable levels, demonstrating an excellent compliance. As there are no established cut-off levels for cortisol following this weight adjusted very low dose DST nor any established cut-offs for any DSTs more generally concerning the identification of hypo- and hypercortisolism, we used the first (10\%) and ninth decile (90\%) of the post-dexamethasone cortisol levels among the controls to define the borders for an abnormally low post-DST cortisol level (also denoted hypersuppression or hypocortisolism) and an abnormally high post-DST cortisol level (also denoted hyposuppression or hypercortisolism), respectively. This translated into a cutoff for hypersuppression of $130 \mathrm{nmol} \mathrm{L}^{-1}$, and a cutoff for hyposuppression of $500 \mathrm{nmol} \mathrm{L}^{-1}$ (Tables 2-6).

\section{Questionnaires}

The Gastrointestinal Symptom Rating Scale-IBS (GSRS-IBS) is a validated self-assessment instrument used to assess symptoms of IBS. $^{41}$ The questionnaire includes thirteen 7-point Likert items grouped into 5 symptom clusters; abdominal pain 2 items), bloating (3 items), constipation ( 2 items), diarrhea ( 4 items), and satiety (2 items). As there is no established sum score cutoff using the scale for finding IBS cases, we decided to use the median sum score of

Table 1. General Characteristics of Study Participants

\begin{tabular}{lccc}
\hline & $\begin{array}{c}\text { Patients } \\
(\mathrm{n}=73)\end{array}$ & $\begin{array}{c}\text { Controls } \\
(\mathrm{n}=146)\end{array}$ & $P$-values \\
\hline Age (mean [SD]), yr & $64.8(14.0)$ & $61.9(9.4)$ & 0.067 \\
Women (n [\%]) & $43(59)$ & $86(59)$ & $>0.99$ \\
BMI (mean [SD]), kg/m ${ }^{2}$ & $26.2(3.7)$ & $25.8(3.7)$ & 0.380 \\
HADS score (mean [SD]) & $10.0(8.1)^{\mathrm{a}}$ & $6.0(5.1)$ & 0.001 \\
GSRS-IBS score (mean [SD]) & $10.0(12.8)^{\mathrm{a}}$ & $6.0(9.0)$ & 0.008 \\
Post-dexamethasone cortisol (mean [SD]), $\mathrm{nmol} \mathrm{L}^{-1}$ & $308(169)$ & $309(141)$ & 0.960 \\
\hline
\end{tabular}

${ }^{\mathrm{a}} \mathrm{n}=61$.

SD, standard deviation; BMI, body mass index; HADS, hospital anxiety and depression scale; GSRS-IBS, gastrointestinal symptom rating scale-irritable bowel syndrome. Significance testing was performed using Student's $t$ test and Pearson's Chi-square test. 
Table 2. Comparing Patients with and Without Functional Gastrointestinal Symptoms Against Controls Concerning the Degree of Hypothalamus-Pituitary-Adrenal Axis Suppression Post-dexamethasone Suppression Test

\begin{tabular}{|c|c|c|c|c|}
\hline & \multicolumn{3}{|c|}{ HPA-axis suppression post-DST } & \multirow[b]{2}{*}{$\begin{array}{c}P \text {-values }{ }^{\mathrm{a}} \\
\text { (vs controls) }\end{array}$} \\
\hline & $\begin{array}{l}\text { Hypersuppressors } \\
\text { (cortisol } \\
\left.<130 \mathrm{nmol} \mathrm{L}^{-1}\right)\end{array}$ & $\begin{array}{c}\text { Intermediate } \\
(\text { cortisol } \\
\left.130-500 \mathrm{nmol} \mathrm{L}^{-1}\right)\end{array}$ & $\begin{array}{c}\text { Hyposuppressors } \\
\quad(\text { cortisol } \\
\left.>500 \mathrm{nmol} \mathrm{L}^{-1}\right)\end{array}$ & \\
\hline Controls $(\mathrm{n}=146, \mathrm{n}[\%])$ & $14(10)$ & $118(80)$ & $14(10)$ & \\
\hline Patients with functional GI symptoms $(\mathrm{n}=30, \mathrm{n}[\%])^{\mathrm{b}}$ & $8(27)$ & $18(60)$ & $4(13)$ & 0.023 \\
\hline Patients without functional GI symptoms $(\mathrm{n}=31, \mathrm{n}[\%])^{\mathrm{b}}$ & $4(13)$ & $22(71)$ & $5(16)$ & 0.370 \\
\hline
\end{tabular}

${ }^{a}$ The 2 analyses are comparisons concerning the categorized post-dexamethasone suppression test (DST) cortisol distribution between the patients with functional gastrointestinal (GI) symptoms and the controls as well as between the patients without functional GI symptoms and controls.

${ }^{b}$ Patients with and without functional GI symptoms were defined as those patients with a gastrointestinal symptom rating scale-irritable bowel syndrome (GSRS-IBS) score above or below the median score for the whole patient sample.

HPA, hypothalamus-pituitary-adrenal; IBS, irritable bowel syndrome.

Significance testing performed with Fisher's exact test. The lowest $10 \%(\mathrm{n}=14)$ and the highest $10 \%$ cutoffs $(\mathrm{n}=14)$ of the post-DST cortisol values $(<130$ $\mathrm{nmol} \mathrm{L} \mathrm{L}^{-1}$ and $>500 \mathrm{nmol} \mathrm{L}^{-1}$ respectively) among the controls were defined as hyper- and hyposuppression respectively and the remaining $80 \%(\mathrm{n}=118)$ were defined as intermediate HPA-axis suppression.

Table 3. Odds Ratios Between Major Depression Patients with Functional Gastrointestinal Symptoms ${ }^{\mathrm{a}}(\mathrm{n}=30)$ and Controls $(\mathrm{n}=146) \mathrm{Used}$ as Reference

\begin{tabular}{lcc}
\hline & $\begin{array}{c}\text { Unadjusted model } \\
\text { OR }(95 \% \text { CI })\end{array}$ & $\begin{array}{c}\text { Adjusted model }^{\mathrm{b}} \\
\text { OR (95\% CI) }\end{array}$ \\
\hline Age $\geq 65 \mathrm{yr}($ reference: $<65 \mathrm{yr})$ & $1.30(0.58-2.89)$ & $1.57(0.60-4.12)$ \\
Male gender $($ reference: female) & $0.72(0.31-1.64)$ & $0.46(0.16-1.27)$ \\
BMI $\geq 25$ (reference: $\mathrm{BMI}<25)$ & $1.39(0.60-3.21)$ & $0.99(0.34-2.83)$ \\
HADS score & $1.20(1.12-1.30)$ & $1.24(1.14-1.35)$ \\
Being a hypersuppressor $^{c}$ (reference: $\left.>130 \mathrm{nmol} \mathrm{L}^{-1}\right)$ & $3.42(1.28-9.13)$ & $7.25(1.97-26.7)$ \\
\hline
\end{tabular}

${ }^{\mathrm{a}}$ Patients with a gastrointestinal symptom rating scale-irritable bowel syndrome (GSRS-IBS) score above median among the patients.

${ }^{\mathrm{b}}$ Odds ratios adjusted for age, gender, body mass index (BMI), hospital anxiety and depression scale (HADS) score, and post- dexamethasone suppression test (DST) cortisol using logistic regression.

${ }^{c} \mathrm{~A}$ subject exhibiting a post-DST cortisol value below $130 \mathrm{nmol} \mathrm{L}{ }^{-1}$ which is the cutoff for the lowest decile (10\%) of the post-DST cortisol values among the controls.

HPA, hypothalamus-pituitary-adrenal; CI, confidence interval.

the patients as the cutoff for functional GI symptomatology. Hence, for a patient to have functional GI symptoms simply means that he scored above the median of the patient sample on GSRS-IBS questionnaire and a patient without functional GI symptoms is a patient who had a score below the median. In order to obtain additional information, we used 5 questions from the former GSRS ${ }^{42}$ concerning symptoms of gastroesophageal reflux (2 items), nausea, vomiting and sucking sensation ( 1 item each), as these symptom clusters are not addressed by the GSRS-IBS. When categorized the specific GI symptoms clusters they were all similar divided by the respective medians of the patient sample and the 2 formed subgroups with respect to each symptom cluster were denoted the "high" and the "low" subgroups respectively (Table 4).

The Hospital Anxiety and Depression Scale (HADS), devel- oped by Zigmond and Snaith in 1983, is a highly sensitive instrument originally designed to screen for symptoms of anxiety and depression among patients with somatic disease. ${ }^{43}$ It consists of Seven 4-point Likert items each for anxiety and depression. We used the HADS because it exhibits high sensitivity in detecting symptoms of anxiety and depression, is thoroughly validated, is easy to fill in, and we have used it in previous research addressing the relationship between anxiety and depression with GI symptoms. ${ }^{44}$ All controls and 61 out of the 73 patients filled out the questionnaires.

\section{Medical Records}

With permission from the participants of the study, medical records from primary care and the clinics of infectious diseases and surgery (including the endoscopy unit) were checked for exclusion 
Table 4. Comparing Patients with High and Low Scores on 7 Gastrointestinal Symptom Clusters Against Controls Concerning the Degree of Hypothalamus-Pituitary-Adrenal Axis Suppression Post-dexamethasone Suppression Test

\begin{tabular}{|c|c|c|c|c|}
\hline & \multicolumn{3}{|c|}{ HPA-axis suppression post-DST } & \multirow{2}{*}{$\begin{array}{c}P \text {-values }{ }^{\mathrm{a}} \\
\text { (vs controls) }\end{array}$} \\
\hline & $\begin{array}{c}\text { Hypersuppressors } \\
\left(\text { cortisol }<130 \mathrm{nmol} \mathrm{L}^{-1} \text { ) }\right.\end{array}$ & $\begin{array}{c}\text { Intermediate } \\
\text { (cortisol 130-500 nmol L }{ }^{-1} \text { ) }\end{array}$ & $\begin{array}{c}\text { Hyposuppressors } \\
\left(\text { cortisol }>500 \mathrm{nmol} \mathrm{L}^{-1} \text { ) }\right.\end{array}$ & \\
\hline Controls ( $\mathrm{n}=146, \mathrm{n}[\%])$ & $14(10)$ & $118(180)$ & $14(10)$ & \\
\hline \multicolumn{5}{|l|}{ Abdominal pain ${ }^{\mathrm{b}}$} \\
\hline $\operatorname{High}(\mathrm{n}=28, \mathrm{n}[\%])$ & $7(23)$ & $17(61)$ & $4(14)$ & 0.036 \\
\hline Low $(\mathrm{n}=33, \mathrm{n}[\%])$ & $5(15)$ & $23(70)$ & $5(15)$ & 0.390 \\
\hline \multicolumn{5}{|l|}{ Bloating $^{\mathrm{b}}$} \\
\hline $\operatorname{High}(\mathrm{n}=23, \mathrm{n}[\%])$ & $7(30)$ & $14(61)$ & $2(9)$ & 0.028 \\
\hline Low $(\mathrm{n}=38, \mathrm{n}[\%])$ & $5(13)$ & $26(68)$ & $7(19)$ & 0.200 \\
\hline \multicolumn{5}{|l|}{ Constipation $^{\mathrm{b}}$} \\
\hline $\operatorname{High}(\mathrm{n}=30, \mathrm{n}[\%])$ & $9(30)$ & $18(60)$ & $3(10)$ & 0.023 \\
\hline Low $(\mathrm{n}=31, \mathrm{n}[\%])$ & $3(10)$ & $22(71)$ & $6(19)$ & 0.250 \\
\hline \multicolumn{5}{|l|}{ Diarrhea $^{\mathrm{b}}$} \\
\hline $\operatorname{High}(\mathrm{n}=33, \mathrm{n}[\%])$ & $8(24)$ & $20(61)$ & $5(15)$ & 0.035 \\
\hline Low $(\mathrm{n}=28, \mathrm{n}[\%])$ & $4(14)$ & $20(72)$ & $4(14)$ & 0.490 \\
\hline \multicolumn{5}{|l|}{ Early Satiety ${ }^{\mathrm{b}}$} \\
\hline $\operatorname{High}(\mathrm{n}=22, \mathrm{n}[\%])$ & $5(23)$ & $15(68)$ & $2(9)$ & 0.220 \\
\hline Low $(\mathrm{n}=39, \mathrm{n}[\%])$ & $7(18)$ & $25(64)$ & $7(18)$ & 0.067 \\
\hline \multicolumn{5}{|l|}{ Nausea $^{\mathrm{b}, \mathrm{c}}$} \\
\hline $\operatorname{High}(\mathrm{n}=23, \mathrm{n}[\%])$ & $6(26)$ & $14(61)$ & $3(13)$ & 0.041 \\
\hline Low $(\mathrm{n}=37, \mathrm{n}[\%])$ & $6(16)$ & $26(70)$ & $5(14)$ & 0.320 \\
\hline \multicolumn{5}{|l|}{ Reflux ${ }^{\mathrm{b}, \mathrm{c}}$} \\
\hline $\operatorname{High}(\mathrm{n}=26, \mathrm{n}[\%])$ & $7(27)$ & $17(65)$ & $2(8)$ & 0.062 \\
\hline Low $(\mathrm{n}=34, \mathrm{n}[\%])$ & $5(15)$ & $23(68)$ & $6(17)$ & 0.200 \\
\hline
\end{tabular}

${ }^{a}$ All analyses are comparisons concerning the categorized post-DST cortisol distributions between the patient subgroups and the controls.

${ }^{b}$ The patients were divided based on the median scores for each specific symptom cluster within the patient sample. Abdominal pain, bloating, constipation, diarrhea, and early satiety were based on items from gastrointestinal symptom rating scale-irritable bowel syndrome (GSRS-IBS). Nausea and reflux were based on items from part of the GSRS. Sixty-one and 60 patients filled in GSRS-IBS and the part of GSRS respectively. The reason for the uneven and varying number of patients within the high and low groups was due to a varying number of patients sharing the exact same scores concerning the different symptom clusters.

'The Nausea cluster includes vomiting and a sucking sensation.

The control consisted of 146 subjects. The lowest $10 \%(\mathrm{n}=14)$ and the highest $10 \%$ cutoffs $(\mathrm{n}=14)$ of the post-dexamethasone suppression test $(\mathrm{DST})$ cortisol values ( $<130 \mathrm{nmol} \mathrm{L}{ }^{-1}$ and $>500 \mathrm{nmol} \mathrm{L}{ }^{-1}$ respectively) within the control sample were defined as hyper- and hyposuppression respectively and the remaining $80 \%$ (n $=118$ ) were defined as having an intermediate hypothalamus-pituitary-adrenal (HPA) axis suppression.

Significance testing performed with Fisher's exact test.

criteria over a period of 6 months covering the study (see above). As a second step with respect to the patients only, we also identified consulters for functional GI disorder and pain conditions in primary care over a 10-year period covering the study. Consulters for functional GI disorder were defined either by diagnosis established by their general practitioner or symptoms according to ROME III criteria. ${ }^{45}$ The Swedish health care system includes a primary care health system with general practioners that take care of all initially referrals (except emergencies). Therefore, all patients who attend the gastroenterology outpatient clinic at Norrland's University Hospital in Umeå are referred from the primary care by a general practitioner. Concerning consulters for pain we decided to focus on 3 frequent conditions, clusters of pain; lumbago, neck pain, and chronic multi-focal pain. Consulters for chronic somatic pain within multiple areas of the body were defined as subjects experiencing pain for more than 6 months within 2 or more areas of the body. The body was categorized into 10 areas: head, neck, thoracic, lumbar spine, shoulder, arm, hand, hip, knee, and foot. Out of the 73 patients the medical records of 70 and 68 cases were deemed of sufficient quality throughout the 10 -year period concerning consulters for functional bowel disorder and pain conditions respectively (Tables 5 and 6). 
Table 5. Comparing Patients Grouped Based on Consulting Behavior for Functional Gastrointestinal Disorder Against Controls Concerning the Degree of Hypothalamus-Pituitary-Adrenal Axis Suppression Post-dexamethasone Suppression Test

\begin{tabular}{|c|c|c|c|c|}
\hline & \multicolumn{3}{|c|}{ HPA-axis suppression post-DST } & \multirow[b]{2}{*}{$\begin{array}{c}P \text {-values }{ }^{\mathrm{a}} \\
\text { (vs controls) }\end{array}$} \\
\hline & $\begin{array}{l}\text { Hypersuppressors } \\
\text { (cortisol } \\
\left.<130 \mathrm{nmol} \mathrm{L}^{-1}\right)\end{array}$ & $\begin{array}{c}\text { Intermediate } \\
(\text { cortisol } \\
\left.130-500 \mathrm{nmol} \mathrm{L}^{-1}\right)\end{array}$ & $\begin{array}{l}\text { Hyposuppressors } \\
\quad(\text { cortisol } \\
\left.>500 \mathrm{nmol} \mathrm{L}^{-1}\right)\end{array}$ & \\
\hline Controls (n = 146, n [\%]) & $14(10)$ & $118(80)$ & $14(10)$ & \\
\hline $\begin{array}{l}\text { Patients who were consulters for functional } \\
\text { GI disorder }(\mathrm{n}=25, \mathrm{n}[\%])^{\mathrm{b}}\end{array}$ & $6(24)$ & $15(60)$ & $4(16)$ & 0.039 \\
\hline $\begin{array}{l}\text { Patients who were non-consulters for functional } \\
\text { GI disorder }(\mathrm{n}=45, \mathrm{n}[\%])^{\mathrm{b}}\end{array}$ & $7(16)$ & $33(73)$ & $5(11)$ & 0.480 \\
\hline
\end{tabular}

${ }^{a}$ All analyses are comparisons concerning the categorized post-dexamethasone suppression test (DST) cortisol distributions between the patient consulters for functional gastrointestinal (GI) disorder and and the controls as well as between the patient non-consulters for functional GI disorder and the controls.

${ }^{b}$ Out of the 73 patients the medical records of 70 cases were deemed of sufficient quality concerning screening for consulters for functional bowel disorder.

The control consisted of 146 subjects. The lowest $10 \%(n=14)$ and the highest $10 \%$ cutoffs $(n=14)$ of the post-DST cortisol values $\left(<130 \mathrm{nmol} \mathrm{L}^{-1}\right.$ and $>500$ $\mathrm{nmol} \mathrm{L} \mathrm{L}^{-1}$ respectively) within the control sample were defined as hyper- and hyposuppression respectively and the remaining $80 \%(\mathrm{n}=118)$ were defined as having an intermediate hypothalamus-pituitary-adrenal (HPA) axis suppression.

Significance testing performed with Fisher's exact test.

\section{Statistical Methods}

Non-parametric tests were used for comparing ordinal scales and continous variables (Mann-Whitney U test) and for performing correlation analyses (Spearman's rank correlation). Fisher's exact test and Pearson's Chi-square test were used for testing distributions of categorical data, and logistic regression was applied when adjusting for possible confounders. There is support that age, gender, body mass index (BMI), and depression/anxiety can influence serum cortisol and were therefore used as covariates. ${ }^{46,47}$ After logit analyses, BMI and age were found to be inappropriate for being used as continous variables within the logistic regression analyses and were therefore dichotomized (cutoffs 65 years and BMI 25). The HADS was treated as a continuous variable. $P$-values below 0.05 were considered significant. All statistical analyses were performed using SPSS 18.0 (SPSS Inc, Chicago, IL, USA).

\section{Results}

\section{Characteristics of Patients and Controls}

The general characteristics common to both patients and controls are shown in Table 1. The strict inclusion criteria of the patients resulted in a sample with a high mean age of 64.8 years. The patients had higher total HADS scores $(P=0.001)$ and higher total GSRS-IBS scores $(P=0.008)$ in comparison with the control subjects, whereas there were no significant differences in age, sex, BMI, or the post-DST cortisol levels. All controls and 61 out of the
73 patients filled out the GSRS and HADS questionnaires. The 12 patients who did not fill out the questionnaires had the following characteristics: mean age 65.2 years (SD 15.8), mean BMI of 27.5 $\mathrm{kg} / \mathrm{m}^{2}$ (SD 4.75), mean post-DST serum cortisol level of $205 \mathrm{nmol}$ $\mathrm{L}^{-1}$ (SD 205), and 8 were women.

\section{The Relationship Between Hypothalamus-Pituitary- Adrenal Axis Function and the Gastrointestinal Symptom Rating Scale-Irritable Bowel Syndrome Score Among Patients}

There was no significant correlation between the total GSRSIBS score and the post-DST serum cortisol level among the patients $\left(r_{s}=-0.156, P=0.230\right)$. However, patients with functional GI symptoms (GSRS-IBS scores above median within the patient sample) were more frequently hypersuppressors compared with the control subjects $(P=0.023$, Table 2$)$. There was no significant difference between the patients without functional GI symptoms (GSRS-IBS scores below median within the patient sample) and the controls with respect to the distribution of hyper- and hyposuppressors $(P=0.370)$ (Table 2). There were no differences in the number of prescription drugs taken $(P=0.570)$ or type of prescription drugs between the patients with functional GI symptoms and patients without, except for selective serotonin reuptake inhibitors (SSRIs) which were more frequent among patients with functional GI symptoms $(P=0.048)$. Patients with functional GI symptoms also had higher HADS scores compared with patients without functional GI symptoms $(P<0.001)$. 
Table 6. Comparing Patients Grouped Based on Consulting Behavior for Pain Conditions Against Controls Concerning the Degree of Hypothalamus-Pituitary-Adrenal Axis Suppression Post-dexamethasone Suppression Test

\begin{tabular}{|c|c|c|c|c|}
\hline & \multicolumn{3}{|c|}{ HPA-axis suppression post-DST } & \multirow[b]{2}{*}{$\begin{array}{c}P \text {-values }{ }^{\mathrm{a}} \\
\text { (vs controls) }\end{array}$} \\
\hline & $\begin{array}{l}\text { Hypersuppressors } \\
\text { (cortisol } \\
<130 \mathrm{nmol} \mathrm{L}^{-1} \text { ) }\end{array}$ & $\begin{array}{c}\text { Intermediate } \\
(\text { cortisol } \\
\left.130-500 \mathrm{nmol} \mathrm{L}^{-1}\right)\end{array}$ & $\begin{array}{c}\text { Hyposuppressors } \\
(\text { cortisol } \\
\left.>500 \mathrm{nmol} \mathrm{L}^{-1}\right)\end{array}$ & \\
\hline Controls $(\mathrm{n}=146, \mathrm{n}[\%])$ & $14(10)$ & $118(80)$ & $14(10)$ & \\
\hline $\begin{array}{l}\text { Patients who were consulters } \\
\text { for lumbago }(\mathrm{n}=19, \mathrm{n}[\%])^{\mathrm{b}}\end{array}$ & $7(37)$ & $12(63)$ & $0(0)$ & 0.006 \\
\hline $\begin{array}{l}\text { Patients who were non-consulters } \\
\text { for lumbago }(\mathrm{n}=49, \mathrm{n}[\%])^{\mathrm{b}}\end{array}$ & $6(12)$ & $35(71)$ & $8(16)$ & 0.320 \\
\hline $\begin{array}{l}\text { Patients who were consulters } \\
\text { for neck pain }(\mathrm{n}=13, \mathrm{n}[\%])^{\mathrm{b}}\end{array}$ & $3(23)$ & $9(69)$ & $1(8)$ & 0.250 \\
\hline $\begin{array}{l}\text { Patients who were non-consulters } \\
\text { for neck pain }(\mathrm{n}=55, \mathrm{n}[\%])^{\mathrm{b}}\end{array}$ & $10(18)$ & $38(69)$ & $7(13)$ & 0.170 \\
\hline $\begin{array}{l}\text { Patients who were consulters } \\
\text { for chronic multifocal pain }(\mathrm{n}=27, \mathrm{n}[\%])^{\mathrm{b}}\end{array}$ & $7(26)$ & $19(70)$ & $1(4)$ & 0.057 \\
\hline $\begin{array}{l}\text { Patients who were non-consulters } \\
\text { for chronic multifocal pain }(\mathrm{n}=41, \mathrm{n}[\%])^{\mathrm{b}}\end{array}$ & $6(15)$ & $28(68)$ & $7(17)$ & 0.200 \\
\hline
\end{tabular}

${ }^{a}$ All analyses are comparisons concerning the categorized post-DST cortisol distributions between each patient subgroups and the control sample.

${ }^{b}$ Out of the 73 patients the medical records of 68 cases were deemed of sufficient quality concerning screening for consulters of pain conditions.

The controls consisted of 146 subjects. The lowest $10 \%(\mathrm{n}=14)$ and the highest $10 \%$ cutoffs $(\mathrm{n}=14)$ of the post-dexamethasone suppression test (DST) cortisol values ( $<130 \mathrm{nmol} \mathrm{L}^{-1}$ and $>500 \mathrm{nmol} \mathrm{L}{ }^{-1}$ respectively) within the control sample were defined as hyper- and hyposuppression respectively and the remaining $80 \%$ (n $=118$ ) were defined as having an intermediate hypothalamus-pituitary-adrenal (HPA) axis suppression.

Significance testing performed with Fisher's exact test.

Applying logistic regression, hypersuppression of the HPAaxis still significantly predicted patients with functional GI symptoms compared with controls with an odds ratio of 7.25 (CI, 1.9726.7) when age, gender, BMI and HADS score were adjusted for (Table 3). This result also remained after further adjustment for usage of SSRIs. The degree of HPA-axis suppression did not predict patients without functional GI symptoms over controls adjusted for the same covariates (adjusted odds ratio 1.57; CI, 0.44-5.55).

\section{The Relationship Between Specific Gastrointestinal Symptoms and Hypothalamus-Pituitary-Adrenal Axis Function Among Patients}

Analyzing each of the 7 GI symptom clusters separately based on the GSRS-IBS questionnaire and questions from part of GSRS, patients with high scores (above median score for the specific cluster within the patient sample) on constipation, bloating, diarrhea, abdominal pain, and dyspepsia were all significantly more often hypersuppressors compared with the controls ( $10 \%$ of the controls were defined as being hypersuppressors) (Table 4). Among the patients with high scores on early satiety and reflux there were no significant differences in the distribution of hyper- and hyposuppressors compared with controls. Patients with low scores concerning the symp- tom clusters (below median score for the specific cluster within the patient sample) did not differ from controls regarding their HPAaxis function (Table 4).

\section{The Relationship Between Hypothalamus-Pituitary- Adrenal Axis Function and Consulting Behavior Among Patients}

Twenty-five patients with unipolar depression had during a 10year period consulted primary care at least once for a functional GI disorder and 4 patients had consulted for gastroesophagal reflux. Patients who had consulted primary care for functional GI disorders were significantly more often hypersuppressors compared with controls ( $10 \%$ of the controls were defined as being hypersuppressors, $P=0.039$ ) (Table 5). There was no difference in the distribution of hyper- and hyposuppressors among patient who were nonconsulters compared with the controls (Table 5). A significantly disproportionate number of the patients who consulted for lumbago were hypersuppressors compared with controls $(P=0.006$ ) (Table 6). Also, patients who consulted primary care for chronic pain within multiple areas of the body were more frequently hypersuppressors compared with the controls $(P=0.057)$ whereas nonconsulters concerning lumbago and multifocal chronic pain did 
not differ with respect to their HPA-axis function compared with controls (Table 6).

\section{Discussion}

The pathophysiology of GI symptoms in patients with affective disorders is poorly understood. We aimed for the first time to investigate the relationship between GI symptoms and the function of the HPA-axis, more specifically the negative feedback response, employing a very low dose DST in patients with major depression. Our main findings are that among the major depression patients there are significant associations between functional GI symptoms based on self-report questionnaires and abnormally low cortisol levels post a low dose DST (ie, HPA-axis hypersuppression), which in a recent review was concluded to be the most common and early characteristic of hypocortisolism. ${ }^{19}$ These associations were further validated by our finding that patients exhibiting HPA-axis hypersuppression were more likely to visit primary care for functional GI disorders. We also found that other common somatic conditions of uncertain etiology exhibited the same pattern since patient hypersuppressors also consulted primary care more frequently for pain conditions such as lumbago and chronic multifocal pain.

Although studies in depression typically have reported the existence of a hypercortisolemic state or hypercortisolism, ${ }^{12-14}$ the existence of a subgroup of depression patients exhibiting hypocortisolism has recently been recognized in patient samples consisting of older outpatients. ${ }^{15-18}$ Our patient sample similarly consisted of older outpatients (mean age 64.8), and our results support that the depression sample was associated with hypocortisolism since the frequency of the hypersuppressor phenotype was increased in our patient sample compared with controls (13/73 [18\%] vs 14/146 [10\%]). We have recently shown in a larger study which contained a major depression patient sample consisting of older outpatients (partly overlapping with the patient sample of the present study) which employed the same DST as the one presently used, that major depression patients exhibited both a significantly higher fraction of subjects with an increased negative feedback response compared with controls and the degree of suppression among patient hypersuppressors was more pronounced compared with the control hypersuppressors. ${ }^{39}$ The study also confirmed that both patient hypersuppressors and control hypersuppressors exhibited significantly lower basal state cortisol levels providing further support to the claim that an elevated negative feedback response of the HPAaxis is associated with a hypocortisolemic state. ${ }^{39}$ Low basal cortisol levels have together with low post-DST cortisol levels, as captured by a low dose DST, as well as a reduced adrenocortiocal reactivity upon challenge (eg, corticotrophin releasing hormone $[\mathrm{CRH}]$ or adrenocorticotropic hormone $[\mathrm{ACTH}]$ administration) all been recognized as characteristics of hypocortisolism, but one reason why the basal cortisol measure is considered a less reliable marker for hypocortisolism could be the large degree of intra-individual variability observed between the circadian cycles, from day to day. The post-DST cortisol value on the other hand is more reflective of the longterm activity level of the HPA-axis. Another reason as to why lowered cortisol levels, post a low dose DST, could be the most reliable characteristic of hypocortisolism is that the measure not only taps into the negative feedback sensitivity, which is adapted to the longterm setting of the circulating cortisol levels, but it also reflects the current basal cortisol level. This, since a low dose DST only moderately lowers the cortisol level and hence should be recognized as a composite measure also reflecting, in part the basal cortisol level upon dexamethasone challenge. Although it has been shown in multiple studies that the post-DST measure, employing a low dose DST, is related the basal cortisol level we still confirmed that the post-DST cortisol measure was correlated to the basal cortisol measure (measured between 8-10 AM prior to the DST) within the patient and the general population samples, also within the current study $(r=0.530 ; P<001$ in patients and $r=0.450 ; P<0.001$ for controls).

Now there is still the questions of which low dose DST that is preferable when trying to identify hypocortisolism as well as which cut-offs to employ. Exactly which individual DST that one decides to opt for is of lesser importance as long as the dexamethasone dose administered is not so high as to cause a cortisol "bottom effect," masking subjects with hypersuppression among subjects with normal suppression. Any DST employed with dexamethasone doses of $0.5 \mathrm{mg}$ or below (such as the DST currently being used) do not suffer from this effect. We have for example prior to the present study employed the currently used very low dose DST in order to identify hypocortisolism exhibited in bipolar disorder and major depression patients as well as in the general population and in all instances the hypersuppressor phenotype was associated with clinically relevant features. Concerning which cut-off of the post-DST cortisol measure that one should adopt, there simply are no established cut-offs as yet. The research on the phenomenon of hypocortisolism have for the most part been quite recently performed and there are as yet no fixed, absolute values pertaining to the HPA-axis characteristics, mentioned above, which are part of the characterization of hypocortisolism and hence to speak of relative hypocortisolism could be considered appropriate and more informative. Since there 
are no established cut-offs for cortisol pertaining to the very low dose DST employed generally, nor are there any established cutoffs for any DSTs concerning the identification of hypocortisolism we decided to denote or identify individuals as hypersuppressors if they exhibited post-DST cortisol values which were relatively lower than the 10th percentile among the controls and this relative hypersuppression is argued to be a characteristic and a marker of hypocortisolism. Regardless of whether one considers this relative hypersuppressor phenotype as a very good marker of hypocortisolism (which we believe) or not, the finding that the phenotype was significantly associated with a cluster of clinically relevant symptoms and conditions remains, is highly interesting and warrants further research.

There are several studies which in an indirect manner corroborate the associations discovered within the present study between depression, functional somatic symptoms such as functional GI symptoms and pain and a hypoactive HPA-axis. For example, in IBS which exhibits a high degree of comorbidity with depression and anxiety disorders, ${ }^{5,6}$ lowered secretion of ACTH and cortisol to a $\mathrm{CRH}$ challenge, lowered evening saliva cortisol and a lowered level of daytime ACTH secretion have been described. ${ }^{9-11}$ Patients with functional somatic syndromes show negative correlations between morning saliva cortisol and depressive symptoms ${ }^{48,49}$ and patients with major depression show a negative correlation between cortisol and somatoform dissociation. ${ }^{50}$ Depressed patients with a comorbid somatoform disorder show an attenuated HPA-axis response to a combined dexamethasone/CRH test. ${ }^{51}$ An enhanced negative feedback sensitivity of the HPA-axis has also been demonstrated in unexplained syndromes associated with pain which show comorbidity with depression. For example, patients with fibromyalgia, ${ }^{28,29}$ chronic myogenous facial pain, ${ }^{20}$ and chronic whiplash-associated disorder ${ }^{33}$ exhibit lowered post-DST cortisol levels. Finally, both burn-out and chronic fatigue syndrome, which are associated with increased depressive symptomatology, have also been reported to exhibit hypoactivity of the HPA-axis. ${ }^{22-26}$

What then could the mechanistic basis, being it causal or noncausal by nature for the observed relationships between the hypersuppressor phenotype in major depression and GI symptoms as well as musculoskeletal pain? Visceral hypersensitivity and visceral hypervigilance are believed to be important factors in the pathophysiology of functional GI disorders. ${ }^{52}$ Lowered cortisol levels and hypoactivity of the HPA-axis could be a marker for a lowered activity within the central $\mathrm{CRH}$ and/or noradrenaline systems. ${ }^{53} \mathrm{~A}$ deficiency in noradrenaline and dopamine signaling has been associated to a lowered activity of the endogenous pain modulatory system and this might play a role in the pathophysiology of hypersensitivity and pain in depression ${ }^{54}$ as well as in IBS and other functional somatic disorders. ${ }^{55}$ It is however also warranted to think that a disturbance centrally within the HPA-axis leading to a relative deficiency of cortisol could be causally responsible for the symptoms and disorders under investigation. Since the HPA-axis with cortisol as its main effector constituent is perhaps our most important long term response system to stress, a relative deficiency of cortisol and stress coping resources could both be risk factors for developing and maintaining depression. Cortisol also has important inhibitory effects upon inflammation, and the presence of a hypocortisolemic state, with increased risk of subtle inflammation, could be the cause for the increased risk of GI symptoms and musculoskeletal pain. This is supported by studies reporting that patients with functional GI disorders, fibromyalgia, and chronic pain conditions more frequently exhibit subtle inflammation. ${ }^{52,56,57}$ This is also supported by a recent study of ours focusing on older outpatients suffering from major depression and a sample representative of the general population which employed the same DST as the present study. The study showed that the hypersuppressor phenotype within the general population sample was significantly associated with elevated C-reactive protein (a nonspecific marker of inflammation) levels (we lacked data concerning $\mathrm{C}$-reactive protein levels for the major depression sample). ${ }^{39}$ We could also show that the hypersuppressor phenotype was associated with shorter leukocyte telomere length, indicative of an increased longterm leukocyte cell turn-over and chronic inflammation in both the major depression and the general population samples. ${ }^{39}$ Chronic pain induced either by disturbances within the pain modulatory system or due to inflammation (or through some other route) can become a significant longterm stressor leading to further exhaustion of the stress-coping resources with subsequent exacerbation of the depressive, GI as well as pain symptoms. For example, it has been shown that the chronicity of stressors is associated with the chronicity of depressive episodes. ${ }^{58,59}$ Finally, another possible explanation for the associations between the HPAaxis function and the bowel symptoms could be factors emanating from the bowel (eg, microbiotica and subtle inflammation) which potentially could influence both the HPA-axis function as well as the mood. Recent studies suggest an associaton between the gut microbiotica and symptoms of anxiety/depression, indicating the complexity pertaining the issue of the brain-gut axis. ${ }^{60}$ Our study do not address the temporal relationships between GI symptoms and depression due to its cross-sectional design and hence we cannot discern whether these relationships are causal or non-causal associations by nature, and if potentially causal we could not differentiate 
between whether the functional GI symptoms are the effects or the causes of a disturbed HPA-axis function.

One limitation of the study is that among the patients where the use of prescription drugs was higher than among the controls the study is underpowered to rule out all possible effects due to drug interactions which potentially could influence GI symptoms. However, we found no difference between the number or type of prescription drugs between the patients with and without functional GI symptoms except for the use of SSRIs. However the result that being a hypersuppressor significantly predicted patients with IBSlike symptomatology did not change after use of SSRIs was added to the logistic regression model (Table 3).

The hypersuppressor phenotype is an aberrant, distinct, and easily detectable phenotype within a biologically fundamental regulatory system which is associated with a cluster of disorders and symptoms, ie, depression, functional GI symptomatology and pain conditions. Our discovery can aid future research aimed at understanding the mechanistic bases of each of these symptoms and disorders separately as well as shed light on how they are connected. More studies are required concerning the reported cluster of symptoms and conditions, addressing HPA-axis hypoactivity, for example, in IBS patients samples while concomitantly also investigating comorbid depressive and pain symptoms, as well as studies on pain conditions taking into account affective and GI comorbidity. Such research could also lead to improved and novel treatment regimens. The most straightforward question would be whether the symptoms in the subgroup of patients exhibiting hypersuppression would respond favorably towards glucocorticoid replacement therapy. Since hypersuppression of cortisol, post a low dose DST, has in a recent review been concluded to be the earliest and most common characteristic of a hypocortisolism the phenotype could become a valuable biomarker also for other clinically significant symptoms and disorders associated with stress-dysregulation.

In summary, we found for the first time that functional GI symptoms as well as consulting behavior for functional GI disorder and pain conditions were all associated with an abnormally increased negative feedback sensitivity of the HPA-axis in patients suffering from major depression.

Acknowledgements: Research Nurses Eva Lundberg, Annelie Nordin, and Lotta Kronberg are thankfully acknowledged for their help and expertise.

Financial support: The research was funded by the Swedish Research Council (Grant No. 2006-4472 and 2009-5269), the
Medical Faculty, Umeå University and the County Councils of Västerbotten and Norrbotten, Sweden.

\section{Conflicts of interest: None.}

Author contributions: Pontus Karling, Rolf Adolfsson, and Karl-Fredrik Norrback designed the research and were involved in the data collection; and Pontus Karling, Mikael Wikgren, Rolf Adolfsson, and Karl-Fredrik Norrback analyzed the data and wrote the paper.

\section{References}

1. Masand P, Kaplan D, Gupta S, et al. Major depression and irritable bowel syndrome: is there a relationship? J Clin Psychiatry 1995;56:363367.

2. Karling P, Danielsson A, Adolfsson R, Norrback KF. No difference in symptoms of irritable bowel syndrome between healthy subjects and patients with recurrent depression in remission. Neurogastroenterol Motil 2007;19:896-904.

3. Corruble E, Guelfi JD. Pain complaints in depressed inpatients. Psychopathology 2000;33:307-309.

4. Fava M. Depression with physical symptoms: treating to remission. J Clin Psychiatry 2003;64(suppl 7):24-28.

5. Wu JC. Psychological co-morbidity in functional gastrointestinal disorders: epidemiology, mechanisms and management. J Neurogastroenterol Motil 2012;18:13-18.

6. Garakani A, Win T, Virk S. Gupta S, Kaplan D, Masand PS. Comorbidity of irritable bowel syndrome in psychiatric patients: a review. Am J Ther 2003;10:61-67.

7. Karling P, Nyhlin H, Wiklund U, Sjöberg M, Olofsson BO, Bjerle P. Spectral analysis of heart rate variability in patients with irritable bowel syndrome. Scand J Gastroenterol 1998;33:572-576.

8. Mayer EA. The neurobiology of stress and gastrointestinal disease. Gut $2000 ; 47: 861-869$

9. Fukudo S, Nomura T, Hongo M. Impact of corticotropin-releasing hormone on gastrointestinal motility and adrenocorticotropic hormone in normal controls and in patients with irritable bowel syndrome. Gut 1998:42:845-849.

10. Dinan TG, Quigley EM, Ahmed SM, et al. Hypothalamic-pituitarygut axis dysregulation in irritable bowel syndrome: plasma cytokines as a potential biomarker? Gastroenterology 2006;130:304-311.

11. Bohmelt AH, Nater UM, Franke S, Hellhammer DH, Ehlert U. Basal and stimulated hypothalamic-pituitary-adrenal axis activity in patients with functional gastrointestinal disorders and healthy controls. Psychosom Med 2005;67:288-294

12. Carroll BJ. The dexamethasone suppression test for melancholia. $\mathrm{Br} \mathrm{J}$ Psychiatry 1982;140:292-304.

13. Holsboer F. The corticosteroid receptor hypothesis of depression. Neuropsychopharmacology 2000:23:477-501.

14. Pariante CM, Miller AH. Glucocorticoid receptors in major depression: relevance to pathophysiology and treatment. Biol Psychiatry 2001;49:391- 
404.

15. Bremmer MA, Deeg DJ, Beekman AT, Penninx BW, Lips P, Hoogendijk WJ. Major depression in late life is associated with both hypo- and hypercortisolemia. Biol Psychiatry 2007;62:479-486.

16. Morrison MF, Redei E, TenHave T, et al. Dehydroepiandrosterone sulfate and psychiatric measures in a frail, elderly residential care population. Biol Psychiatry 2000;47:144-150.

17. Oldehinkel AJ, van den Berg MD, Flentge F, Bouhuys AL, ter Horst GJ, Ormel J. Urinary free cortisol excretion in elderly persons with minor and major depression. Psychiatry Res 2001;104:39-47.

18. Penninx BW, Beekman AT, Bandinelli S, et al. Late-life depressive symptoms are associated with both hyperactivity and hypoactivity of the hypothalamo-pituitary-adrenal axis. Am J Geriatr Psychiatry 2007;15:522529.

19. Fries E, Hesse J, Hellhammer J, Hellhammer DH. A new view on hypocortisolism. Psychoneuroendocrinology 2005;30:1010-1016.

20. Galli U, Gaab J, Ettlin DA, Ruggia F, Ehlert U, Palla S. Enhanced negative feedback sensitivity of the hypothalamus-pituitary-adrenal axis in chronic myogenous facial pain. Eur J Pain 2009;13:600-605.

21. Heim C, Ehlert U, Hellhammer DH. The potential role of hypocortisolism in the pathophysiology of stress-related bodily disorders. Psychoneuroendocrinology 2000;25:1-35.

22. Papadopoulos A, Ebrecht M, Roberts AD, Poon L, Rohleder N, Cleare AJ. Glucocorticoid receptor mediated negative feedback in chronic fatigue syndrome using the low dose $(0.5 \mathrm{mg})$ dexamethasone suppression test. J Affect Disord 2009;112:289-294.

23. Cleare AJ. The neuroendocrinology of chronic fatigue syndrome. Endocr Rev 2003;24:236-252.

24. Roberts AD, Wessely S, Chalder T, Papadopoulos A, Cleare AJ. Salivary cortisol response to awakening in chronic fatigue syndrome. Br J Psychiatry 2004;184:136-141.

25. Kudielka BM, Bellingrath S, Hellhammer DH. Cortisol in burnout and vital exhaustion: an overview. G Ital Med Lav Ergon 2006;28(suppl 1): 34-42.

26. Pruessner JC, Hellhammer DH, Kirschbaum C. Burnout, perceived stress, and cortisol responses to awakening. Psychosom Med 1999;61:197-204.

27. Rydmark I, Wahlberg K, Ghatan PH, et al. Neuroendocrine, cognitive and structural imaging characteristics of women on longterm sickleave with job stress-induced depression. Biol Psychiatry 2006;60:867-873.

28. Riva R, Mork PJ, Westgaard RH, Rø M, Lundberg U. Fibromyalgia syndrome is associated with hypocortisolism. Int $\mathrm{J}$ Behav Med 2010;17:223-233.

29. Wingenfeld K, Wagner D, Schmidt I, Meinlschmidt G, Hellhammer $\mathrm{DH}, \mathrm{Heim} \mathrm{C}$. The low-dose dexamethasone suppression test in fibromyalgia. J Psychosom Res 2007;62:85-91

30. Yehuda R. Status of glucocorticoid alterations in post-traumatic stress disorder. Ann NY Acad Sci 2009;1179:56-69.

31. Yehuda R, Southwick SM, Krystal JH, Bremner D, Charney DS, Mason JW. Enhanced suppression of cortisol following dexamethasone administration in posttraumatic stress disorder. Am J Psychiatry 1993;150:83-86.
32. Sudhaus S, Fricke B, Stachon A, et al. Salivary cortisol and psychological mechanisms in patients with acute versus chronic low back pain. Psychoneuroendocrinology 2009;34:513-522.

33. Gaab J, Baumann S, Budnoik A, Gmünder H, Hottinger N, Ehlert $\mathrm{U}$. Reduced reactivity and enhanced negative feedback sensitivity of the hypothalamus-pituitary-adreanal axis in chronic whiplash-associated disorder. Pain 2005;119:219-224.

34. McBeth J, Chiu YH, Silman AJ, et al. Hypothalamic-pituitary-adrenal stress axis function and the relationship with chronic widespread pain and its antecedents. Arthritis Res Ther 2005;7:R992-R1000.

35. Miller GE, Chen E, Zhou ES. If it goes up, must it come down? Chronic stress and the hypothalamic-pituitary-adrenocortical axis in humans. Psychol Bull 2007;133:25-45.

36. American Psychiatric Association. Diagnostic and statistical manual of mental disorders. 4th ed. Washington, DC: APA 1994.

37. Nilsson LG, Bäckman L, Erngrund K, et al. The Betula prospective cohort study: memory, health, and aging. Aging Neuropsychol Cogn 1997;4:1-32.

38. Rask E, Olsson T, Söderberg S, et al. Tissue-specific dysregulation of cortisol metabolism in human obesity. J Clin Endocrinol Metab 2001;86:1418-1421.

39. Wikgren M, Maripuu M, Karlsson T, et al. Short telomeres in depression and the general population are associated with a hypocortisolemic state. Biol Psychiatry 2012;71:294-300.

40. Maripuu M, Wikgren M, Karling P, Adolfsson R, Norrback KF. Relative hypo- and hypercortisolism are both associated with depression and lower quality of life in bipolar disorder: a cross-sectional study. PLoS One 2014; 9:e98682.

41. Wiklund IK, Fullerton S, Hawkey CJ, et al. An irritable bowel syndrome-specific symptom questionnaire: development and validation. Scand J Gastroenterol 2003;38:947-954.

42. Dimenäs E, Glise H, Hallerbäck B, Hernqvist H, Svedlund J, Wiklund I. Quality of life in patients with upper gastrointestinal symptoms. An improved evaluation of treatment regimens? Scand J Gastroenterol 1993;28:681-687.

43. Zigmond AS, Snaith RP. The hospital nxiety and depression scale. Acta Psychiatr Scand 1983;67:361-370.

44. Bjelland I, Dahl AA, Haug TT, Neckelmann D. The validity of the hospital anxiety and depression scale. An update literature review. J Psychosom Res 2002;52:69-77.

45. Thompson WG, Longstreth GF, Drossman DA, Heaton KW, Irvine EJ, Müller-Lissner SA. Functional bowel disorders and functional abdominal pain. Gut 1999;45(suppl 2):II43-II47.

46. Claes SJ. CRH, stress, and major depression: a psychobiological interplay. Vitam Horm 2004;69:117-150.

47. Salehi M, Ferenczi A, Zumoff B. Obesity and cortisol status. Horm Metab Res 2005;37:193-197.

48. Mutsuura H, Kanbara K, Fukunaga M, et al. Depression and anxiety correlate differently with salivary free cortisol in the morning in patients with functional somatic syndrome. Appl Psychophysiol Biofeedback 2009;34:291-298.

49. Bob P, Freybergh P, Jasova D, et al. Depression, cortisol and somatoform 
dissociative symptoms. Neuro Endocrinol Lett 2008;29:235-239.

50. Bob P, Fedor-Freybergh P, Jasova D, et al. Dissociative symptoms and neuroendocrine dysregulation in depression. Med Sci Monit 2008;14:CR499-CR504.

51. Veen G, DeRijk RH, Giltay EJ, van Vliet IM, van Pelt J, Zitman FG. The influence of psychiatric comorbidity on the dexamethason/CRH test in major depression. Eur Neuropsychopharmacol 2009; 19:409-415.

52. Rodríguez-Fandiño O, Hernández-Ruiz J, Schmulson M. From cytokines to toll-like receptors and beyond - current knowledge and future research needs in irritable bowel syndrome. J Neurogastroenterol Motil 2010;16:363-373.

53. Gold PW, Chrousos GP. Organization of the stress system and its dysregulation in melancholic and atypical depression: high vs low $\mathrm{CRH} / \mathrm{NE}$ states. Mol Psychiatry 2002;7:254-275.

54. Stahl S, Briley M. Understanding pain in depression. Hum Psychopharmacol 2004;19(suppl 1):S9-S13.
55. Mayer EA, Naliboff BD, Chang L. Basic pathophysiologic mechanisms in irritable bowel syndrome. Dig Dis 2001;19:212-218.

56. Törnblom H, Abrahamsson H, Barbara G, et al. Inflammation as a cause of functional bowel disorders. Scand J Gastroenterol 2005;40:11401148.

57. Maletic V, Raison CL. Neurobiology of depression, fibromyalgia and neuropathic pain. Front Biosci 2009;14:5291-5338.

58. Keller MB, Shapiro RW, Lavori PW, Wolfe N. Relapse in major depressive disorder: analysis with life table. Arch Gen Psychiatry 1982;39:911915.

59. Kendler KS, Kessler RC, Walters EE, et al. Stressful life events, genetic liability, and onset of major depression in women. Am J Psychiatry 1995;152:833-842.

60. Zhou L, Foster JA. Psychobiotics and the gut-brain axis: in pursuit of happiness. Neuropsychiatr Dis Treat 2015;16:715-723. 